\title{
TAVR versus surgery in low-risk patients
}

The success
of these two
trials is likely
to change the
way aortic
valve disease
is treated,
particularly
among younger
patients

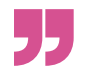

Transcatheter aortic valve replacement (TAVR) was initially developed $>1$ decade ago for elderly patients with severe aortic stenosis who were at very high or prohibitive surgical risk, but international guidelines have since expanded its use to include patients at intermediate risk, given trial data showing that TAVR was noninferior to surgical aortic valve replacement in this patient group. Now, two new clinical trials report that TAVR with a balloonexpandable valve or a self-expanding valve is at least as safe and effective as surgical aortic valve replacement in patients at low surgical risk. "The next step towards studying truly lowrisk patients was a small one given the fact that so far, no long-term penalties have been seen," comments Nicolas M. Van Mieghem from Erasmus University Rotterdam, Netherlands, who was not part of the trials. "[In addition], the TAVR technique has evolved even further towards a therapy [that is performed] under local anaesthesia, with a short stay at the intensive care unit and early discharge opportunities." Results from the two trials were presented at the ACC.19 Scientific Sessions in New Orleans, and simultaneously published in the NEJM.

The Evolut Low Risk trial was a multicentre, randomized,

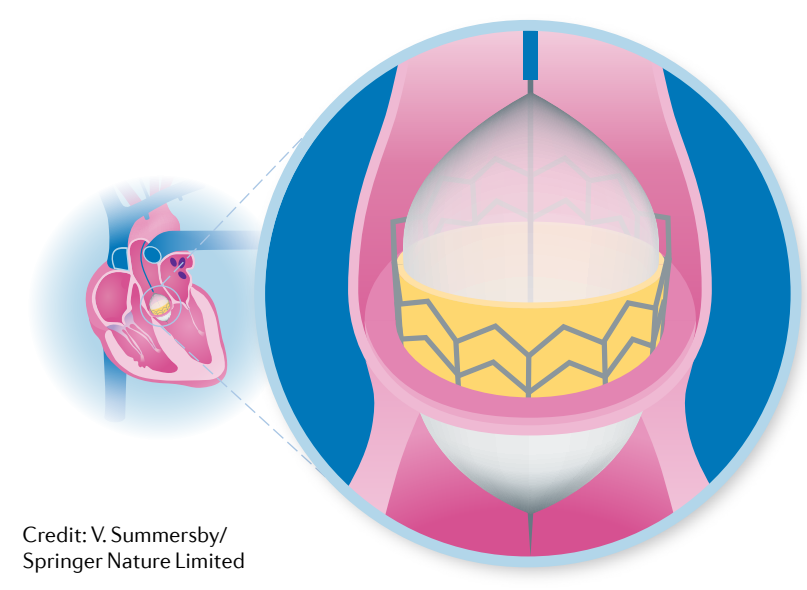

noninferiority trial designed to assess the safety and effectiveness of TAVR with a self-expanding bioprosthesis compared with surgical aortic valve replacement in patients with severe aortic valve stenosis and $\leq 3 \%$ risk of death by 30 days with surgery. In total, 1,403 patients underwent TAVR $(n=725)$ or surgery $(n=678)$. The primary end point of death or disabling stroke at 24 months occurred in 5.3\% (95\% Bayesian credible interval (BCI) 3.3-8.0) of the TAVR group and $6.7 \%$ (95\% BCI 4.4-9.6) of the surgery group. The prespecified criterion for noninferiority was met (posterior probability $>0.999$ ), but the requirement for superiority was not (posterior probability $=0.779$ ). The incidence of the 30-day composite safety end point was $5.3 \%$ in the TAVR group and $10.7 \%$ in the surgery group. Furthermore, at 30 days, patients who had undergone TAVR, as compared with surgery, had a lower incidence of atrial fibrillation (7.7\% versus $35.4 \%$ ) and life-threatening bleeding (2.4\% versus $7.5 \%$ ), and a higher incidence of moderate or severe aortic regurgitation (3.5\% versus $0.5 \%)$. At 12 months, the TAVR group had lower mean aortic valve gradients than the surgery group (8.6 $\mathrm{mmHg}$ versus $11.2 \mathrm{mmHg}$ ).

"Definitive conclusions regarding the advantages and disadvantages of TAVR as compared with surgery await long-term clinical and echocardiographic follow-up," state the researchers. All patients in the trial will be followed up for 10 years.

Investigators in the multicentre, randomized PARTNER 3 trial similarly sought to compare the safety and efficacy of TAVR versus surgery in patients with severe aortic stenosis at low operative risk, but performed TAVR using a third-generation balloon-expandable valve. Recruited patients were randomly assigned to undergo either TAVR ( $n=503)$ or surgery $(n=497)$. The composite end point of death from any cause, stroke or rehospitalization at 1 year was significantly lower in the TAVR group than in the surgery group ( $8.5 \%$ versus $15.1 \%$, absolute difference -6.6 percentage points, 95\% CI -10.8 to $-2.5, P<0.001$ for noninferiority; HR 0.54 , 95\% CI $0.37-0.79, P=0.001$ for superiority). The incidence of several key secondary end points was also significantly lower in the TAVR group than in the surgery group, including new-onset atrial fibrillation at 30 days $(5.0 \%$ versus $39.5 \% ; P<0.001)$, death or stroke at 30 days $(1.0 \%$ versus $3.3 \% ; P=0.01$ ) and length of hospitalization ( 3 days versus 7 days; $P<0.001$ ). Furthermore, TAVR was associated with faster improvements in NYHA class and 6-min walking test distance compared with surgery. The majority of the 30-day safety end points were similar between the two groups.

A major limitation of this trial is the short duration of follow-up. "We don't know the durability of these valves, so all patients will be followed up for 10 years," explains Michael J. Mack, the lead investigator of PARTNER 3. Although the Evolut Low Risk and the PARTNER 3 trials had different clinical end points, they show that TAVR is at least equivalent to surgery in terms of efficacy and safety for low-risk patients with severe aortic stenosis. The success of these two trials is likely to change the way aortic valve disease is treated, particularly among younger patients.

Karina Huynh

ORIGINAL ARTICLES Popma, J. J. et al.

Transcatheter aortic-valve replacement with a self-expanding valve in low-risk patients. N. Engl. J. Med. https://doi.org/10.1056/NEJMoa1816885 (2019) | Mack, M. J. et al. Transcatheter aortic valve replacement with a balloon-expandable valve in low-risk patients. N. Engl.J.Med. https://doi.org/ 10.1056/NEJMoa1814052 (2019) FURTHER READING Puri, R. et al. Future of transcatheter aortic valve implantation - evolving clinical indications. Nat. Rev. Cardiol. 15, 57-65 (2018) 